INPLASY

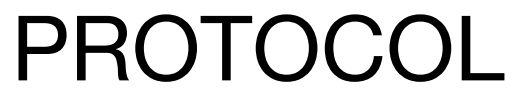

To cite: Luo et al. Tobacco smoke exposure and risk of different pathologic types of stroke: A comprehensive systematic review and doseresponse meta-analysis. Inplasy protocol 202060062. doi:

10.37766/inplasy2020.6.0062

Received: 17 June 2020

Published: 17 June 2020

Corresponding author: Li-Ming Lu

lulimingleon@126.com

Author Affiliation:

Guangzhou University of

Chinese Medicine

Support: 2020B1111100008; 2019QNPY02

Review Stage at time of this submission: Preliminary searches.

Conflicts of interest:

None.

\section{Tobacco smoke exposure and risk of different pathologic types of stroke: A comprehensive systematic review and dose-response meta-analysis}

\author{
Luo, J1; Tang, X2; Wen, H33 Tang, C4; Lu, L5; Xu, N6.
}

Review question / Objective: What is the impact of smoking on stroke, and is there any difference in stroke morbidity and mortality. What are the effects on incidence and mortality in four subtypes of stroke(ischemic stroke, hemorrhagic stroke, intracerebral hemorrhage (ICH) and subarachnoid hemorrhage (SAH) respectively)? Do dose and cessation of smoking influence stroke risk?

Condition being studied: Smoking, incidence and mortality of stroke, ischemic stroke, hemorrhagic stroke, intracerebral hemorrhage and subarachnoid hemorrhage.

INPLASY registration number: This protocol was registered with the International Platform of Registered Systematic Review and Meta-Analysis Protocols (INPLASY) on 17 June 2020 and was last updated on 04 February 2021 (registration number INPLASY202060062).

\section{INTRODUCTION}

Review question / Objective: What is the impact of smoking on stroke, and is there any difference in stroke morbidity and mortality. What are the effects on incidence and mortality in four subtypes of stroke(ischemic stroke, hemorrhagic stroke, intracerebral hemorrhage $(\mathrm{ICH})$ and subarachnoid hemorrhage (SAH) respectively)? Do dose and cessation of smoking influence stroke risk?

Condition being studied: Smoking, incidence and mortality of stroke, ischemic stroke, hemorrhagic stroke, intracerebral hemorrhage and subarachnoid hemorrhage. 


\section{METHODS}

Search strategy: This study will include participants who were included in prospective observational studies about smoking and stroke.

Participant or population: This study will include participants who were included in prospective observational studies about smoking and stroke.

Intervention: This study is meta-analysis without any intervention.

Comparator: We will compare the participants's risk of stroke according to their smoking status, including never and ever smoking (former smoker and current smoker).

Study designs to be included: Prospective observational studies on smoke and stroke will be included.

Eligibility criteria: This study will include all prospective observational studies that compared the risk of stroke according the smoking status of participants.

Information sources: In this meta-analysis, we systematically searched the Books@Ovid, Journals@Ovid, Your Journals@Ovid, Joanna Briggs Institute EBP Database -, ACP Journal Club, CCTR, CDSR, CCA, CLCMR, DARE, CLHTA, CLEED, AMED, Embase, Ovid Emcare, HAPI, HealthSTAR, Ovid MEDLINE(R) by using Ovid for English language studies until January 31, 2021. In addition, we manually searched for extra relevant articles in the reference lists of identified articles and other publications.

Main outcome(s): The primary outcomes are differences in the risk of stroke morbidity and mortality between smokers and non-smokers.

Additional outcome(s): The secondary outcomes are the risk of incidence and mortality in four subtypes of stroke and the influence of smoking dose and cessation to stroke risk. We will perform dose-response meta-analysis to exact dose-risk and quitting duration-risk relationships between smoke and stroke.

Data management: Data and relevant information were extracted from the eligible studies by two authors (LJY and TXR). Basic information about authors, year of publication, inclusion and exclusion criteria, sample size, consisting of population (age, gender, countries and continents and whether suffer from cardiovascular disease or relevant diseases at baseline), definition of smoking, classification of smoking status(current, former, never, dose of smoking expose, duration of smoking cessation), multiple-adjusted OR, HR or RR with $95 \%$ Cls of stroke for each smoking status category, follow-up time were extracted in a standard table.

Quality assessment / Risk of bias analysis: We will evaluate quality of included studies using the Newcastle-Ottawa Scale (NOS) for cohort studies. Each study will be judged on the basis of a 9-point scale using three broad criteria: selection of participants (4 points), comparability of studies ( 2 points), and ascertainment of outcome of interest (3 points). High study quality will be defined as a score of 7 or greater, moderate quality as 3 to 6 points, and low quality as below 3 points. If there are disagreements in the data extraction and quality assessment between the two authors, a third author will be consulted.

Strategy of data synthesis: In this metaanalysis, we systematically searched the PubMed, Embase, and other databases by using Ovid for English language studies until January 31, 2021. Search terms included words associated with stroke and the Cochrane Tobacco Addiction Group search strategy.

Subgroup analysis: If data are available, a subgroup analysis will be performed to detect sources of obvious heterogeneity. Subgroup analysis also will be performed to search the difference between current smoker and former smoker versus never smoker. 
Sensibility analysis: Leave-one-out sensitivity analysis will be conducted for the primary outcome. We also will conduct sensitivity analysis by excluding non-highquality literature.

Language: English.

Country(ies) involved: China.

Keywords: stroke, incidence and mortality, smoking, meta-analysis.

Contributions of each author:

Author 1 - Jian-Yu Luo - Author 1 design search strategy, screen identified articles, extract and analyze data, assessment quality, draft and revise manuscripts.

Author 2 - Xiao-Rong Tang - Author 2 screen identified articles, extract and analyze data, assessment quality, draft manuscripts.

Author 3 - Hao Wen - Author 3 analyse date.

Author 4 - Chun-Zhi Tang - Author 4 (Corresponding author) design and direct research, revise manuscripts.

Author 5 - Li-Ming Lu - Author 5 (Corresponding author) design and direct research, revise manuscripts.

Author 6 - Neng-Gui Xu - Author 6 (Corresponding author) design and direct research, revise manuscripts. 